\title{
A NON SYMMETRICAL VALUE FOR GAMES WITHOUT TRANSFERABLE UTILITIES; APPLICATION TO REINSURANCE*
}

\author{
JEAN LEMAIRE
}

\begin{abstract}
We define axiomatically a concept of value for games without transferable utilities, without introducing the usual symmetry axiom. The model-a generalization of a previous paper [6] extending Nash's bargaining problem-attempts to take into account the affinities between the players, defined by an a priori set of "distances". The general solution of all three- and four-person games is described, and various examples are discussed, like the classical "Me and my Aunt" and a reinsurance model.
\end{abstract}

Nous définissons de manière axiomatique un concept de valeur pour les jeux à utilités non-transférables, sans introduire l'axiome classique de symétrie. Le modèle - une généralisation d'un concept de valeur [6] étendant à plusieurs joueurs le problème de marchandage de Nash — tient compte des affinités entre les joueurs, données sous forme d'une matrice de "distances" a priori. Nous donnons la solution générale de tous les jeux à trois et quatre joueurs, et discutons plusieurs exemples classiques, dont le célèbre "Ma tante et moi" et le modèle de réassurance de Borch.

\section{INTRODUCTION}

In most of the value concepts of the cooperative theory of games [6], [10], [12], the authors have enforced a symmetry axiom: every symmetrical game has a symmetrical solution; that is, if the characteristic function of the game is symmetrical with respect to the bissecting line passing through the initial payoffs, the solution grants the same utility increase to each player. If this axiom seems innocuous (it is evident that the final payoff must not depend on a permutation, on a re-numbering of the players), it implies the implicit assumptions that the game is adequately represented by the characteristic function and that no element outside this function influences the behaviour of the participants and the results of the game. But everyday observations suggest that the players usually do not behave as one would expect from the abstract study of the game: some coalitions are formed more easily than others, two players that should coalize in order to make a profit do not unite because of personal antipathy, some persons are more likely to enter in a coalition with a given group than others, etc...; the characteristic function form of the game seems unable to forecast the coalitions that will effectively form, since it does not take into account the personal affinities between the players. For instance, the French Communist party, during the Fourth Republic consistently the largest party, never managed to enter into a government coalition, because no other party was ever willing to join it in a coalition.

* Presented at the $14^{\text {th }}$ ASTIN Colloquium, Taormina, October 1978. 
So the value-say the Shapley value, or any value computed on the basis of the characteristic function only-of this party is largely overestimated, since it does not consider the aversion of the other parties.

We shall in this paper develop a value concept that attempts to catch the notion of "affinities", by suppressing the symmetry axiom and introducing "distances" between players. It is a modification of our former [6] symmetrical value.

\section{AXIOMS}

Let $[N, v(C), \xi]$ be a game without transferable utilities (shortly a non-transferable game), where

$-N=\{1, \ldots, n\}$ is the set of the $n$ players;

- $v(C)$ is the characteristic function, defined on all the non-void subsets $C$ of $N$ (the coalitions); the image of this function is a subset $v(C)$ of $E^{|C|}$, the Euclidean space of dimension $|C|$, such that $v(C)$ is non-empty, closed, convex and super-additive:

$$
\forall C_{a}, C_{b} \subset N \supset-C_{a} \cap C_{b}=\phi, v\left(C_{a} U C_{b}\right) \supset v\left(C_{a}\right) x v\left(C_{b}\right) ;
$$

$-\xi$ is the prospect space for the grand coalition $N$, i.e. the space delimited by the Pareto-optimal surface $v(N)$ and the hyperplanes perpendicular to the axes whose coordinates are the initial utilities of the players.

Let $\left[C, v\left(C^{\prime}\right), \xi_{C}\right]$ be the subgame associated to the coalition $C$. The purpose of this paper is to define a value for such games. We shall assume that the players will sign a treaty

$$
\bar{y}(N)=\left[y_{1}(N), \ldots, y_{n}(N)\right],
$$

where $y_{j}(N)$ specifies the monetary payoff to player $j$. Since such a treaty usually involves side-payments (whose sum must be zero), the components of $\bar{y}(N)$ must satisfy a linear admissibility condition

$$
y_{1}(N)+\ldots+y_{n}(N)=z
$$

(the model can easily be extended to the games without side-payments. In that case the treaties have to mention the commodities owned or exchanged by each participant).

An example of a non-transferable game is the classical exchange of risks. Let the players be $n$ insurance companies, of respective situations $\left[S_{j}, F_{j}\left(x_{j}\right)\right]$, where $S_{j}$ is the initial surplus of company $j$, and $F_{j}\left(x_{j}\right)$ the distribution function of its total claim amount. Each company evaluates its situation by an utility function 


$$
U_{j}\left(x_{j}\right)=U_{j}\left[S_{j}, F_{j}\left(x_{j}\right)\right]=\int_{0}^{\infty} u_{j}\left(S_{j}-x_{j}\right) d F_{j}\left(x_{j}\right),
$$

where $u_{j}(x)$ is the utility of a monetary amount $x$, with $u_{j}^{\prime}(x) \geqslant 0$ and $u_{j}^{\prime \prime}(x) \leqslant 0$. The members of the pool will try to improve their situations by concluding a treaty of risk exchanges

$$
\bar{y}=\left[y_{1}\left(x_{1}, \ldots, x_{n}\right), \ldots, y_{n}\left(x_{1}, \ldots, x_{n}\right)\right],
$$

where $y_{j}\left(x_{1}, \ldots, x_{n}\right)$ is the amount that $j$ has to pay if the claims for the different companies are respectively $x_{1}, \ldots, x_{n}$.

Since all the claims must be indemnified, the $y_{j}\left(x_{1}, \ldots, x_{n}\right)$ must satisfy the admissibility condition

$$
\sum_{j=1}^{n} y_{j}\left(x_{1}, \ldots, x_{n}\right)=\sum_{j=1}^{n} x_{j}=z
$$

the total amount of all claims. After the signature of $\bar{y}$, the utility of $j$ becomes

$$
U_{j}(\bar{y})=\int_{\theta} u_{j}\left[S_{j}-y_{j}(\bar{x})\right] d F(\bar{x})
$$

where $\theta$ is the positive orthant of $E^{n}$ and $F(\bar{x})$ the $n$-dimensional distribution function of the claims $\bar{x}=\left(x_{1}, \ldots, x_{n}\right)$.

$\bar{y}$ is Pareto-optimal if there is no $\bar{y}^{\prime}$ such that $U_{j}\left(\bar{y}^{\prime}\right) \geqslant U_{j}(\bar{y}) \forall j$, with at least one strict inequality. Borch (see for instance [1]) has demonstrated that all the Pareto-optimal treaties are characterized by the following relations.

$$
k_{j} u_{j}^{\prime}\left[S_{j}-y_{j}(\bar{x})\right]=k_{1} u_{1}^{\prime}\left[S_{1}-y_{1}(\bar{x})\right] \quad k_{j} \geqslant \circ \forall j
$$

Let $K=\left\{k_{1}, \ldots, k_{n}\right\}$. The treaty is unique for given $K$, but there usually exists an infinity of $K$ satisfying $\left(1^{\prime}\right)$ and (2).

It has been shown [5] that this reinsurance market is in fact a non-transferable game and that the problem of selecting an optimal set of constants $k_{f}$ is identical to the determination of the value of the game. In [7] we have computed the Shapley value and the Nash-Lemaire value [6] of this game. Both values use the classical symmetry axiom. In the sequel, we shall extend axiomatically the latter value to the non-symmetrical case. We shall use four axioms.

\section{Axiom 1: Linear invariance}

The solution is not affected by a linear transformation performed on the utilities of the players.

Justification: Since utilities are only defined up to a linear transformation, it must obviously be the case for the solution. 


\section{Axiom 2: Strong Pareto-optimality}

The solution depends on all the sub-treaties relative to all the sub-coalitions (with the exception of the sub-coalitions that form with probability zero-see section 4). Each sub-treaty (and the final treaty) must be Pareto-optimal and satisfy the admissibility condition.

Justification: The axiom expresses the fact that, during a negotiation, the bargaining strength of a player depends on the terms he obtained during the preceding discussions; a player will get more from his partners if he has signed a favourable treaty in a sub-coalition. We thus authorize the formation of any coalition during the bargaining process. Each one may negotiate with a disjoint group in order to unify. During this partial bargain, we suppose that each coalition acts as a single player: no one has the right to disavow his signature and quit his coalition in order to negotiate separately. We also assume that the grand coalition is formed step by step; at each step two coalitions only merge, so that $N$ is obtained after $(n-1)$ steps $\left.{ }^{1}\right)$. Since the power of a player depends on all the already signed contracts, they must influence the final payoff. Each sub-treaty must of course be Pareto-optimal in the corresponding sub-game, and the admissibility condition must be satisfied.

\section{Axion 3: Independence of irrelevant alternatives}

During each negotiation between two coalitions, exclusion from the prospect space of possible payoffs other than the solution and the disagreement point (the utilities that the players get in case they cannot reach an agreement) does not affect the solution.

Justification: This axiom means that the solution, which by axiom 2 must lie on the upper boundary of the prospect space, only depends on the shape of this boundary in its neighbourhood, and not on distant points. This expresses a structure property of the bargaining process: during the negotiations, the set of the alternatives likely to be selected progressively reduces, so that at the end of the discussion, the solution must only compete with very close points, and not with propositions already eliminated during the prior stages of the bargaining.

\section{Axiom 4: Partial symmetry}

If, during a negotiation between two disjoint groups, the prospect space is symmetrical, so must be the treaty signed.

1) Those behavioural hypotheses are not very restrictive since the axiom considers all the grouping possibilities. For instance, we prohibit the simultaneous merging of three disjoint groups $C_{a}, C_{b}, C_{c}$. But the solution will in particular study the grouping of $C_{a}$ and $C_{b}$ at one step and the adjunction of $C_{c}$ during the next step. The two other cases $\left(C_{a}\right.$ and $C_{c}$ unify first then absorb $C_{b}$, and $C_{b}$ and $C_{c}$ group and join $C_{a}$ one step later) will also be considered. In the same fashion, some schemes of coalition forming where one player remains isolated until the final step, will intervene in the final treaty. 
Justification: The classical symmetry axiom is weakened, since we only enforce it for the sets of two players or groups of players. It implies that the affinities between the players do not affect the discussions between two coalitions, which consist of a tough haggling between two groups trying to take as much advantage as they can from the situation. The affinities will intervene in the kind of coalitions that tend to form, in the propensity that some players have to start discussing with a particular group instead of another. In other words, the affinities influence the choice of the groups that enter negotiation, but not their negotiation itself. For example, the recent French political events demonstrate that the fact that the Communists and the Socialists have a strong affinity does not incite them to make concessions to each other: coalition forming and bargaining are two different things.

Therefore, we shall separate the computation of the value of a game in two distinct parts:

1. the coalition forming procedure, which consists of the determination of a set of probabilities $W=\left\{W_{C_{a} \dot{U} \overline{C_{a}}} \forall C \subset N, \forall C_{a} \subset C, \overline{C_{a}}=C \mid C_{a}, C_{a} \neq \phi\right.$, $\left.\overline{C_{a}} \neq \phi\right\}$, interpreted as "weights associated to orders of formation of the coalitions $C=C_{a} U \overline{C_{a} " ;}$

2. the bargaining procedure, which attributes a payoff to each player, given the set $W$.

\section{THE BARGAINING PROCEDURE: EXISTENCE AND UNICITY THEOREM}

Let us denote $\bar{y}(C)=\bar{y}\left(x_{i} \mid i \in C\right)$ the treaty signed by a coalition $C$ and $U_{i}(C)=U_{i}\left[y_{i}(C)\right]$ the utility $i \in C$ derives from this signature.

Suppose that, at a given moment of the negotiation, a first group $C_{a}$ of players has reached an agreement and signed a treaty $\bar{y}\left(C_{a}\right)$, allowing to each of its members an utility $U_{i}\left(C_{a}\right)$, while another group $C_{b}$ (such that $C_{a} \cap C_{b}=$ $\phi$ ) has concluded a treaty $\bar{y}\left(C_{b}\right)$, giving to each $j \in C_{b}$ an utility $U_{j}\left(C_{b}\right)$. Both groups meet in order to conclude a global treaty $\bar{y}\left(C_{a} \dot{U} C_{b}\right.$ ) (the symbol $\dot{U}$ has a slightly different meaning than the usual reunion sign. $C_{a} \dot{U} C_{b}$ means " $C_{a}$ joins $C_{b}$ ". The $\cdot$ is placed to recall that the result not only depends on the set $C_{a} U C_{b}$, but also on the manner in which this coalition was formed, i.e. on $C_{a}$ and $C_{b}$ ). If both coalitions cannot agree on a treaty $\bar{y}\left(C_{a} \dot{U} C_{b}\right)$, they necessarily return to the starting point of the negotiation, awarding to each player $U_{i}\left(C_{a}\right)$ (if $i \in C_{a}$ ) or $U_{j}\left(C_{b}\right)$ (if $j \in C_{b}$ ). For this reason, this point is called the disagreement point.

\section{Lemma:}

There exists one and only one treaty satisfying the axioms. It can be obtained by maximizing the expression 
(3)

$$
\prod_{i \in C_{a}}\left[U_{i}\left(C_{a} \dot{U} C_{b}\right)-U_{i}\left(C_{a}\right)\right] . \underset{j \in C_{b}}{ }\left[U_{j}\left(C_{a} \dot{U} C_{b}\right)-U_{j}\left(C_{b}\right)\right],
$$

providing each term of the product is non-negative.

\section{Proof}

The demonstration is a slight generalization of Nash's result [6]. Denote I the number of players of $C_{a}(0<I<n)$ and $L$ the cardinality of $C_{b}(0<L \leqslant$ $n-I)$. Number the players in such a way that the members of $C_{a}$ occupy the indices 1 to $I$ and the players of $C_{b}$ the indices $I+1$ to $L$. The vector

$$
\bar{U}_{d}=\left[U_{1}\left(C_{a}\right), \ldots, U_{I}\left(C_{a}\right), U_{I+1}\left(C_{b}\right), \ldots, U_{I+L}\left(C_{b}\right)\right]
$$

is the disagreement point of this negotiation. Let $\psi$ be the maximum of (3). $\psi$ is unique because of the convexity of $\xi_{C_{a} U C_{b}}$.

Suppose that $\psi$ is distinct from $\bar{U}_{d}$ (otherwise the problem is trivial since the prospect space consists of a single point). We can subject all the players' utility functions to a linear transformation $\tau$, by changing their origins so as to carry $\bar{U}_{d}$ to $\bar{U}_{d}^{\tau}=(0, \ldots, 0)$ and their units to carry $\psi$ to $\psi^{\tau}=(1, \ldots, 1)$. Let $\xi_{C_{a} U C_{b}}^{\tau}=\tau\left(\xi_{C_{a} U C_{b}}\right)$ be the image of $\xi_{C_{a} U C_{b}}$ by $\tau . \xi_{C U C_{b}}^{\tau}$ is convex. $\psi^{\tau}$ is the unique point of tangency between $\xi_{C_{a} U C_{b}}^{\tau}$ and the hyperboloid whose equation is

$$
\prod_{i=1}^{I+L} U_{i}=1 .
$$

$\xi_{C_{a} U C_{b}}^{\tau}$ is even completely under the hyperplane $H_{1}$ of equation

$$
\sum_{i=1}^{I+L} U_{i}=I+L
$$

In fact, if a point $P \in \xi_{C a U}^{\tau} C_{b}$ was such that $\sum_{i=1}^{I+L} U_{i}>I+L$, it would be the same for any point of the segment $P \psi^{\tau}$ by convexity. Some of the points of this segment would be inside the hyperboloid, with thus $\prod_{i=1}^{I+L} U_{i}>1$, contradicting the fact that $\psi^{\tau}$ maximizes $\prod_{i=1}^{i} U_{i}$.

Under $H_{1}$ we can construct a half hypersphere $\sigma$ around $\psi^{\tau}$ with a radius sufficiently large as to include $\xi_{C_{a} U C_{b}}^{\tau}$. Consider first the game whose prospect space is limited by $\sigma$ and $H_{1}$. This game is symmetrical, and $\psi^{\tau}$ is its solution by axioms 2 and 4 . Axiom 3 allows us to withdraw all the points of $\sigma \mid \xi_{C_{a} U C_{b}}^{\tau}$ without altering the solution. Finally through axiom 1 we can perform the inverse transformation

$$
\xi_{C_{a} U C_{b}}=\tau^{-1}\left(\xi_{C_{a} U C_{b}}^{\tau}\right)
$$

and assert that $\psi=\tau^{-1}\left(\psi^{\tau}\right)$ is the optimal point. 
Note that, as announced in the discussion of axiom 4, the negotiation between two groups of players is a "pure" bargaining, i.e. not influenced by affinities between players.

\section{Theorem:}

To each set of probabilities $W$ can be associated one and only one treaty $\bar{y}(N)$ satisfying all the axioms. It can be obtained by the recursion.

$$
\begin{aligned}
& y_{i}(\{i\})=x_{i} \\
& \vdots \\
& y_{i}(C)=\left\{\begin{array}{l}
\sum_{c_{a}(c} W_{C_{a} \dot{U} \overline{C_{a}}} y_{i}\left(C_{a} \dot{U} \overline{C_{a}}\right) \\
\vdots \\
c_{a} \neq \phi
\end{array}\right. \\
& i \in C\left\{\begin{array}{l}
c=|C| \\
\forall C>-1<c<n \\
\overline{C_{a}}=C \mid C_{a}
\end{array}\right. \\
& i \notin C \\
& y_{i}(N)=\sum_{\substack{C_{a} \subset N \\
C_{a} \neq \phi}} W_{C a \dot{U}} \overline{C_{a}} y_{i}\left(C_{a} \dot{U} \overline{C_{a}}\right) \\
& i=1, \ldots, n \cdot \overline{C_{a}}=N \mid C_{a},
\end{aligned}
$$

(4)

where, at each step, $\underset{\boldsymbol{c}_{a} \subset \boldsymbol{c}}{\sum} W_{C_{a} \dot{\boldsymbol{U}} \overline{C_{a}}}=1$ and $W_{C_{a} \dot{U} C_{a}}>0$, and $y_{i}\left(C_{a} \dot{U} \overline{C_{a}}\right)$ is obtained by maximizing (3), with the disagreement point

$$
\begin{array}{ll}
U_{i}\left(C_{a}\right) & i \in C_{a} \\
U_{j}\left(\overline{C_{a}}\right) & j \in \overline{C_{a}} .
\end{array}
$$

\section{Proof}

1. Existence: It is sufficient to verify that $\bar{y}(N)$ satisfies all the axioms. This proof is straightforward.

2. Suppose that, for a given set $\left\{W_{C_{a} \dot{U} \overrightarrow{C_{a}}}\right\}$, there exist two different optimal solutions $\bar{y}(N)$ and $\bar{y}^{\prime}(N)$, i.e. there exists at least an $i$ such that $y_{i}(N) \neq y_{i}^{\prime}(N)$.

We shall first show that the two solutions must differ in at least a partial treaty. In other words, it is impossible that $y_{i}\left(C_{a} \dot{U} \overline{C_{a}}\right)=y_{i}^{\prime}\left(C_{a} \dot{U} \overline{C_{a}}\right)$ for all $C_{a} \subset N$ and that $y_{i}(N) \neq y_{i}^{\prime}(N)$. (4) expresses that the partial treaties $y_{i}\left(C_{a} \dot{U} \overline{C_{a}}\right)$ are summarized by a weighted arithmetic mean. One could of course think of other parameters, like the geometric or the quadratic mean for instance, but the only parameter satisfying the admissibility condition is the weighted arithmetic mean

$$
y_{i}(N)=\sum_{\substack{C_{a} C_{N} \\ C_{a} \neq \phi}} W_{C_{a} \dot{U} \overline{C_{a}}}^{i} y_{i}\left(C_{a} \dot{U} \overline{C_{a}}\right) .
$$


We shall now show that the admissibility condition also implies that $W_{C_{a} \dot{U} \overline{C_{a}}}^{i}=W_{C_{a} \dot{U} \overline{C_{a}}}^{1} \forall i$. It is sufficient to prove it for $n=3$. In this case, there are only three ways to form the grand coalition, which we shall note to simplify

$$
\begin{aligned}
& A=\{12\} \dot{U}\{3\} \\
& B=\{13\} \dot{U}\{2\} \\
& C=\{23\} \dot{U}\{1\} .
\end{aligned}
$$

Thus $y_{1}(N)=W_{A}^{1} y_{1}(A)+W_{B}^{1} y_{1}(B)+W_{C}^{1} y_{1}(C)$

$y_{2}(N)=W_{A}^{2} y_{2}(A)+W_{B}^{2} y_{2}(B)+W_{C}^{2} y_{2}(C)$

$y_{3}(N)=W_{A}^{3} y_{3}(A)+W_{B}^{3} y_{3}(B)+W_{C}^{3} y_{3}(C)$.

(1) allows us to replace $y_{1}(A)$ by $z-y_{2}(A)-y_{3}(A)$, with similar relations for $y_{1}(B)$ and $y_{1}(C)$. We obtain

$$
\begin{aligned}
& y_{1}(N)=W_{A}^{1}\left[z-y_{2}(A)-y_{3}(A)\right]+W_{B}^{1}\left[z-y_{2}(B)-y_{3}(B)\right]+W_{C}^{1}\left[z-y_{2}(C)-\right. \\
& \left.-y_{3}(C)\right] \\
& y_{2}(N)=W_{A}^{2} y_{2}(A)+W_{B}^{2} y_{2}(B)+W_{C}^{2} y_{2}(C) \\
& y_{3}(N)=W_{A}^{3} y_{3}(A)+W_{B}^{3} y_{3}(B)+W_{C}^{3} y_{3}(C) .
\end{aligned}
$$

Summing, and using (1), we get

$$
\begin{aligned}
z & =y_{2}(A)\left(W_{A}^{2}-W_{A}^{1}\right)+y_{3}(A)\left(W_{A}^{3}-W_{A}^{1}\right)+y_{2}(B)\left(W_{B}^{2}-W_{B}^{1}\right)+ \\
& +y_{3}(B)\left(W_{B}^{3}-W_{B}^{1}\right)+y_{2}(C)\left(W_{C}^{2}-W_{C}^{1}\right)+y_{3}(C)\left(W_{C}^{3}-W_{C}^{1}\right)+ \\
& +W_{A}^{1} z+W_{B}^{1} z+W_{C}^{1} z
\end{aligned}
$$

Since the $W$ 's are the coefficients of a weighted arithmetic mean, $W_{A}^{1}+W_{B}^{1}+W_{C}^{1}=1$, and the sum

$$
\begin{aligned}
& y_{2}(A)\left(W_{A}^{2}-W_{A}^{1}\right)+y_{2}(B)\left(W_{B}^{2}-W_{B}^{1}\right)+y_{2}(C)\left(W_{C}^{2}-W_{C}^{1}\right) \\
+ & y_{3}(A)\left(W_{A}^{3}-W_{A}^{1}\right)+y_{3}(B)\left(W_{B}^{3}-W_{B}^{1}\right)+y_{3}(C)\left(W_{C}^{3}-W_{C}^{1}\right)
\end{aligned}
$$

must be identically equal to zero, $\forall y_{2}$ and $y_{3}$. Thus $W^{i}=W^{1} \forall i$.

So there exists a coalition $C_{a} \subset N$ such that $y_{i}\left(C_{a} \dot{U} \overline{C_{a}}\right) \neq y_{i}^{\prime}\left(C_{a} \dot{U} \overline{C_{a}}\right)$. Since the solution of the maximization of $(3)$ is unique, this result can only be explained by a difference of the disagreement points $y_{i}\left(C_{a}\right)$ and $y_{i}^{\prime}\left(C_{a}\right)$. Suppose $U_{i}\left[y_{i}\left(C_{a}\right)\right]<U_{i}\left[y_{i}^{\prime}\left(C_{a}\right)\right]$. There exists a player $j \in C_{a}$ such that $U_{j}\left[y_{j}\left(C_{a}\right)\right]$ $>U_{j}\left[y_{j}^{\prime}\left(C_{a}\right)\right]$, for otherwise $\bar{y}\left(C_{a}\right)$ would not be Pareto-optimal in the subgame $\left[C_{a}, v\left(C_{a}^{\prime}\right), \xi_{C_{a}}\right]$.

The same argument can be repeated iteratively for the coalition $C_{a}$ : there exists a $C_{b} \subset C_{a}$ such that $U_{i}\left[y_{i}\left(C_{b}\right)\right]<U_{i}\left[y_{i}^{\prime}\left(C_{b}\right)\right] . j$ must also belong to $C_{b}$ (or another player $j^{\prime}$ such that $\left.U_{j^{\prime}}\left[y_{j},\left(C_{b}\right)\right]>U_{j^{\prime}}\left[y_{j}^{\prime},\left(C_{b}\right)\right]\right)$ ), in fact, if $j$ were a member of $C_{a} \mid C_{b}, \bar{y}\left(C_{b}\right)$ would not be Pareto-optimal in $\left[C_{b}, v\left(C_{b}^{\prime}\right), \xi_{C_{b}}\right]$ as $\bar{y}^{\prime}\left(C_{a} / C_{b}\right)$ in $\left[\left.C\right|_{a} C_{b}, v\left(C_{b}^{\prime}\right), \xi_{C_{a} \mid C_{b}}\right]$ and axiom 2 would be violated. 
So we can present a finite succession of coalitions

$$
N \supset C_{a} \supset C_{b} \supset \ldots \supset C_{f} \supset \ldots \supset C_{F}
$$

such that, for all $f<F$ :

$$
\begin{aligned}
& i, j \in C_{f} ; \\
& U_{i}\left[y_{i}\left(C_{f}\right)\right]<U_{i}\left[y_{i}^{\prime}\left(C_{f}\right)\right] ; \\
& U_{j}\left[y_{j}\left(C_{f}\right)\right]>U_{j}\left[y_{j}^{\prime}\left(C_{f}\right)\right] .
\end{aligned}
$$

The last term $C_{F}$ can only be the coalition formed by players $i$ and $j$ (otherwise we could have continued the process). There exists thus two treaties $\bar{y}\left(C_{F}\right)$ and $\bar{y}^{\prime}\left(C_{F}\right)$, Pareto-optimal in $\left[\{i j\}, v(C), \xi_{\{i j\}}\right]$, i.e. such that

$$
\begin{aligned}
& \max \left\{U_{i}\left[y_{i}(\{i, j\})\right]-U_{i}\left[y_{i}(\{i\})\right]\right\} \cdot\left\{U_{j}\left[y_{j}(\{i, j\})\right]-U_{j}\left[y_{j}(\{j\})\right]\right\} \\
= & \max \left\{U_{i}\left[y_{i}^{\prime}(\{i, j\})\right]-U_{i}\left[y_{i}^{\prime}(\{i\})\right]\right\} \cdot\left\{U_{j}\left[y_{j}^{\prime}(\{i, j\})\right]-U_{j}\left[y_{j}^{\prime}(\{j\})\right]\right\} .
\end{aligned}
$$

This contradicts the lemma, applied to the coalitions $C_{a}=\{i\}$ and $C_{b}=\{j\}$.

The solution is constructed by induction on the number of players of the coalitions: one must successively compute the value of all the two-player coalitions, then all the three-player sets, ... to end up finally with the grand coalition. The optimal treaty for a coalition $C$ of $c$ players is obtained by considering the set of its $2^{c-1}-1$ (strict) sub-coalitions $\mathrm{C}_{a}$ for which there already exists a computed sub-treaty. For each $C_{a}$, one computes by (3) a treaty $\bar{y}\left[C_{a} \dot{U}\left(C \backslash C_{a}\right)\right]$. The utility granted to a player never diminishes when one or more partners are added to the coalition: (3) always provides a $U_{i}\left(C_{a} \dot{U} \overline{C_{a}}\right)$ greater or equal than $U_{i}\left(C_{a}\right)$. The higher his disagreement point, the higher the utility awarded to a player. The procedure provides $2^{c-1}-1$ (generally) different partial treaties, which are summed up by a weighted arithmetic mean. The fact that $W_{C_{a}}^{i} \dot{U} \overline{C_{a}}$ does not depend on $i$ allows us to interpret those weights as "probabilities associated to orders of formation of the coalitions".

To sum up, the value concept takes into consideration all the possible orders of formation of the grand coalition, weighted by their respective probabilities; each player allies with other players or sets of players so that after $(n-1)$ junctions $N$ is formed and a treaty concluded. All the grouping possibilities are considered, weighted, and account in the final solution.

For $n=2$, the value coincides with the unweighted value [6], the Nash solution [8] and the Shapley value [12].

For $n=3$, the value weights three different partial treaties $\bar{y}[\{12\} U\{3\}]$, $\bar{y}[\{13\} \dot{U}\{2\}]$ and $\bar{y}[\{1\} \dot{U}\{23\}]$. Since the disagreement points are computed on the basis of coalitions of one or two persons, the partial treaties are the same as in the symmetrical value. The solution differs generally from the Shapley value. 
For $n>3$, however, the generalization is more than just "adding weights" to the partial treaties, since the disagreement points already take the affinities into account and favour the close partners.

Nothing was said up to now as far as the determination of the weights $W_{C_{a} \dot{U} \overline{C_{a}}}$ is concerned. This will be the subject of the next section.

\section{FORMALIZATION OF THE AFFINITY CONCEPT: THE COALITION FORMING}

PROCEDURE

We suppose that the affinity between two players can be expressed by a nonnegative number, $d_{i j}$, representing the "distance" (in a broad sense) between $i$ and $j$ : the larger the distance, the lesser the affinity between both players. $d_{i j}=\infty$ means that the antipathy between them is so strong that they will never join together a sub-coalition $\left.{ }^{2}\right)$. On the other hand, $d_{i j}=0$ implies that the coalition $\{i, j\}$ will immediately form. This is a relatively uninteresting case, since it amounts to the same thing to consider $\{i, j\}$ as a single player. It is therefore not restrictive to suppose that the (symmetrical) matrix of the distances (the figures of the diagonal are irrelevant) does not contain more than one zero in each row or column (the reunion of three players in a single step is indeed not allowed, although the model could be easily adapted to this case, by introducing as a first stage the merging of the three players with probability one).

Define the "distance" between two coalitions $C_{a}$ and $C_{b}$ by

$$
d_{C_{a}, C_{b}}=\frac{\sum_{i \in C_{a}} \sum_{j \in C_{b}} d_{i j}}{\left|C_{a}\right| \cdot\left|C_{b}\right|} .
$$

The value of all the two-player coalitions can easily be computed by (3). Suppose, by induction, that we have already computed the solution for all the sets containing at most $(n-1)$ players. It only remains to calculate the value of the grand coalition.

A coalition configuration of order $m$ (shortly a $m$-configuration) is a vector

$$
C^{m}=\left(C_{1}, \ldots, C_{m}\right) \quad \begin{gathered}
C_{a} \cap C_{b}=\phi \quad a \neq b \\
U_{a=1}^{m} \quad C_{a}=N \\
C_{a} \neq \phi \quad \forall a,
\end{gathered}
$$

$\left.{ }^{2}\right)$ However, the hypotheses of the model imply that they will be forced to cooperate at the final step, since the grand coalition is bound to eventually form. This is a consequence of the fact that we required the value of a $n$-person game, a value that is useless if we know in advance that $N$ will never form. But, as our theory also provides the value of all the $(n-1)$-person subgames, as well as the probabilities of formation of each subcoalition, no modification is required when one (or more) of the distances is infinite. 
indicating the coalitions formed after step $(n-m)$. During a negotiation, $m$ successively takes all the integer values, decreasing from $n$ to 1 . At the beginning, $n=m$, and $C^{n}=(\{1\},\{2\}, \ldots,\{n\})$. After the final junction, $m=1$ and $C^{1}=(\{1 \ldots n\})$. For $1<m<n$ there exists several different coalition configurations, denoted by $C_{x}^{m}, C_{y}^{m}, \ldots$ Let $M_{m}$ be the set of all the $m$-configurations. We shall denote $i \sim j$ if $i$ and $j$ belong to the same coalition of $C^{m}$, $i \frac{1}{\tau} j$ if they do not.

Each $m$-configuration $C^{m}$ generates a number of descendants $C^{m-1}$ obtained by joining two coalitions of $C^{m}$. Let $D_{1}$ be the set of all the descendants of $C^{m}$. Of course, two different $m$-configurations can produce the same descendant. Let $W_{C^{m}}$ be the probability that $C^{m}$ forms during the procedure, and $W_{C^{m-1} \mid C^{m}}$ the (conditional) probability that $C^{m}$ generates $C^{m-1}$.

Naturally, this probability is zero if $C^{m-1}$ cannot be a descendant of $\mathrm{Cm}^{m}$.

We must associate to each distance matrix $D$ a set $W$ of probabilities $W_{C_{a} \dot{U} \overline{C_{a}}}$, defined $\forall C \subset N, \forall C_{a} \subset C \supset-\overline{C_{a}}=C \backslash C_{a}, C_{a} \neq \phi, \overline{C_{a}} \neq \phi$.

$$
D=\left\{d_{i j}\right\} \vec{R} W=\left\{W_{C_{a} \dot{U}} \overline{C_{a}}\right\}
$$

Of course not any rule $R$ that associates a set $W$ to a matrix $D$ is suitable for our problem. A rule will be said coherent if it satisfies the following conditions.

Condition I (Rules of probability calculus)

1.a. $W_{C^{m}} \geqslant 0 \quad \forall C^{m}$

1.b. $\Sigma W_{C^{m}}=1 \quad m=1, \ldots, n$

$M_{m}$

1.c. $\sum_{D_{1}}^{\Sigma} W_{C^{m-1} \mid C^{m}}=1 \quad \forall C^{m}$

1.d. $W_{C^{m-1}}=\sum_{M_{m}} W_{C^{m-1}} \mid C^{m} \cdot W_{C^{m}} \quad \forall C^{m-1}$

Condition 2 (Relation between affinities and probabilities)

2.a. $W_{C^{m}}$ is a non-increasing function of $d_{i j} \quad \forall C^{m} \supset-i \sim j$

$W_{C^{m}}$ is a non-decreasing function of $d_{\vec{i}} \quad \forall C^{m} \supset-i \tau j$

2.b. $\lim _{d_{i j} \rightarrow 0} W_{C^{n-1}}=1 \quad i \sim j$

2.c. $\begin{array}{ll}\lim _{d_{v \rightarrow \infty}} W_{C^{m}}=0 \quad \forall C^{m}, \quad i \sim j \\ & \forall m \supset-\quad 1<m<n\end{array}$

Condition 3 (Possible symmetry of two players)

3. If $d_{j l}=d_{i l} \forall l$, then $W_{C_{x}^{m}}=W_{C_{y}^{m}}$, where $C_{y}^{m}$ is obtained from $C_{x}^{m}$ by commuting $i$ and $j$. 
Condition 4 (Relations between successive configurations)

4. If $W_{C_{x}^{m}}>W_{C_{y}^{m}}$, then $W_{C_{x}^{m-1}}>W_{C_{y}^{m-1}} \forall m$, if $C_{x}^{m-1}$ is a descendant of $C_{x}^{m}$ and if $C_{y}^{m-1}$ is the descendant of $C_{y}^{m}$ obtained through the same adjunction.

Condition 5 (Relations between configuration probabilities and weights)

5. $W_{C a \dot{U} \overline{C_{a}}}=W_{C^{2}}, \quad \forall C_{a}$, where $C^{2}=\left(C_{a}, \overline{C_{a}}\right)$.

Condition 6 (Invariance with respect to a similarity)

6. $W$ is not affected by a multiplication of the distances by a positive constant: if $d_{i j}^{\prime}=k d_{i j} \quad \forall i j, W^{\prime}=W$.

Note that any coherent rule determines a set $W$ whose cardinality exceeds by far (for $n>2$ ) the number of distances. It can be shown that $|D|=$ $\frac{n(n-1)}{2}-1$ and $|W|=\sum_{i=3}^{n}\left(\begin{array}{l}n \\ i\end{array}\right)\left(2^{i-1}-2\right)$.

We obtain the following numbers for $3 \leqslant n \leqslant 10$.

\begin{tabular}{rcr}
\hline$n$ & $\begin{array}{c}\text { Number of } \\
\text { distances }\end{array}$ & $\begin{array}{c}\text { Number of } \\
\text { probabilities }\end{array}$ \\
\hline 3 & 2 & 2 \\
4 & 5 & 14 \\
5 & 9 & 64 \\
6 & 14 & 244 \\
7 & 20 & 846 \\
8 & 27 & 2,778 \\
9 & 35 & 8,828 \\
10 & 44 & 27,488 \\
\hline
\end{tabular}

There exists few coherent rules. In the sequel, we shall use the following rule

$$
W_{C^{m-1} \mid C^{m}}=\frac{\frac{1}{d_{C_{a}}^{2}, C_{b}}}{\sum_{c=1}^{m} \sum_{d=c} \frac{1}{d_{C_{c}}^{2}, C_{d}}}
$$

where $C^{m-1}=\left(C_{1}, \ldots, C_{a} U C_{b}, \ldots, C_{m}\right)$ is the descendant of $C^{m}=\left(C_{1}, \ldots\right.$, $\left.C_{a}, \ldots, C_{b}, \ldots, C_{m}\right)$. We thus suppose the attraction between two coalitions inversely proportional to the square of their distance. 


\section{RESOLUTION SCHEME OF ALL THREE-PERSON GAMES}

1. Suppose three players, 1,2 and 3 , of initial utilities $U_{1}(\{1\}), U_{2}(\{2\})$ and $U_{3}(\{3\})$, and of affinities defined by the set $\left(d_{12}, d_{13}, d_{23}\right)$. For the sake of simplicity, we shall in the sequel omit the braces, e.g. write 12 instead of $\{12\}$.

2. The maximization of the products

$$
\begin{aligned}
& {\left[U_{1}(12)-U_{1}(1)\right] \cdot\left[U_{2}(12)-U_{2}(2)\right]} \\
& {\left[U_{1}(13)-U_{1}(1)\right] \cdot\left[U_{3}(13)-U_{3}(3)\right]} \\
& {\left[U_{2}(23)-U_{2}(2)\right] \cdot\left[U_{3}(23)-U_{3}(3)\right]}
\end{aligned}
$$

provides the treaties

$$
\begin{aligned}
& \bar{y}(12)=\left[y_{1}(12), y_{2}(12)\right] \\
& \bar{y}(13)=\left[y_{1}(13), y_{3}(13)\right] \\
& \bar{y}(23)=\left[y_{2}(23), y_{3}(23)\right] .
\end{aligned}
$$

3. Grand coalition

\begin{tabular}{lllll}
\hline $\begin{array}{l}m \\
m\end{array}$ Configuration & Probability & & \\
\hline $\begin{array}{l}(1,2,3) \\
(12,3) \\
(13,2) \\
(1,23)\end{array}$ & $\begin{array}{l}W_{12,3}=A / d_{12}^{2} \\
W_{13,2}=A / d_{13}^{2}\end{array}$ & where $A=\frac{1}{\frac{1}{d_{12}^{2}}+\frac{1}{d_{13}^{2}}+\frac{1}{d_{23}^{2}}}$ \\
& $W_{1,23}=A / d_{23}^{2}$
\end{tabular}

Example 1. The constant-sum three-person game.

The characteristic function of this game is

$$
\begin{aligned}
& v(\phi)=v(1)=v(2)=v(3)=0 \\
& v(12)=v(13)=v(23)=v(123)=1 .
\end{aligned}
$$


1. Initial utilities

2. 2-player coalitions
Utilities

$$
(.0, \quad .0, \quad .0)
$$

\begin{tabular}{|c|c|c|}
\hline$\left(\begin{array}{l}1 \\
\dot{U}\end{array} 2\right)$ & $(.5$, & .5 , \\
\hline$(1 U 3)$ & $(.5$, & .0 \\
\hline$(2 U 3)$ & $(.0$, & .5 , \\
\hline
\end{tabular}

3. Grand coalition. Distances: $d_{12}=1, d_{13}=2, d_{23}=2.5$

\begin{tabular}{|c|c|c|c|c|}
\hline Formation of $N$ & Probability & & & \\
\hline$\left(12 \dot{U}_{3}\right)$ & $W_{12 \dot{U} 3}=W_{12,3}=.7092$ & $(.5$ & .5 & .0 \\
\hline$\left({ }_{13} \dot{U}_{2}\right)$ & $W_{13 \dot{U} 2}=W_{13,2}=.1773$ & $(.5$ & .0 & .5 \\
\hline \multirow[t]{2}{*}{$\left({ }_{1} \dot{U}_{23}\right)$} & $W_{1 \dot{U} 23}=W_{1,23}=.1135$ & (.o, & .5 & $.5 \quad$ \\
\hline & Value & $(.4433$ & .4113 & .1454 \\
\hline
\end{tabular}

We notice that 1 and 2 take a big advantage of their vicinity. Besides, the solution converges towards $(.5, .5, .0)$ as $d_{12}$ approaches 0.1 becomes a little more than 2 because he is slightly nearer of 3 .

Example 2. A pair of shoes.

" 1 owns a left shoe. 2 and 3 are each in possession of a right shoe. The pair can be sold for 1 unit. How much is 1 entitled to?" This exemple is famous in game theory because important concepts like the core, the bargaining set, the kernel and the nucleolus completely fail to catch the threat possibilities of coalition (23) and lead to the paradoxical allotment $(1,0,0)$. Moreover, the solution is the same if there are 999 left shoes and 1,000 right shoes: the situation becomes nearly symmetrical and the owners of right shoes still get nothing. The Shapley value, $\left(\frac{2}{3}, \frac{1}{6}, \frac{1}{8}\right)$, is certainly more intuitive, although it seems a bit too generous towards 1 . Our unweighted value is $\left(\frac{4}{9}, \frac{5}{18}, \frac{5}{18}\right)$.

The characteristic function is

$$
\begin{aligned}
& v(\phi)=v(1)=v(2)=v(3)=v(23)=0 \\
& v(12)=v(13)=v(123)=1 .
\end{aligned}
$$

\begin{tabular}{|c|c|c|c|c|}
\hline Formation of $N$ & Probability & & Utility & \\
\hline$\left(12 \dot{U}_{3}\right)$ & $W_{12 \dot{U} 3}=.7092$ & $(.5$ & .5 & .0 \\
\hline$\left(13 \dot{U}_{2}\right)$ & $W_{13 \dot{u} 2}=.1773$ & $(.5$ & .0 & .5 \\
\hline \multirow[t]{2}{*}{$\left(1 \dot{U}_{23}\right)$} & $W_{1 \dot{v} 23}=.1135$ & $(.3333$ & .3333 & $.3333)$ \\
\hline & Value & $\left(.4^{811}\right.$, & .3924 & $.1265)$ \\
\hline
\end{tabular}

Using the same distances as in example 1, we obtain 
One notices that 2 makes the most out of his friendship with 1. The solution converges towards $(.5, .5,0)$ as $d_{12} \rightarrow 0$. The share of 1 , always included in the interval $[1 / 3,1 / 2]$, diminishes when 2 and 3 feel more inclined to coalize before entering discussion with him. For the set $\left(d_{12}=2, d_{13}=2.5, d_{23}=1\right)$, for instance, the solution is $\left(.3^{818}, .3252, .2930\right)$. It tends to $(1 / 3,1 / 3,1 / 3)$ as $d_{23} \rightarrow 0$.

Example 3. The reinsurance model.

As Gerber [3], [4] has shown that exponential utility functions possess very desirable properties for insurers, we shall suppose that

$$
u_{j}(x)=\frac{1}{a_{j}}\left(1-e^{-a_{j} x}\right) \quad j=1, \ldots, n .
$$

Solving equations (2), taking into account the admissibility condition ( $\left.\mathbf{1}^{\prime}\right)$, leads to the solution

where

$$
y_{j}(\bar{x})=q_{j} z+y_{j}(\mathrm{o})
$$

$$
q_{j}=\frac{\frac{1}{a_{j}}}{\sum_{i=1}^{n} \frac{1}{a_{i}}}
$$

and

$$
y_{j}(0)=S_{j}-q_{j} \sum_{i=1}^{n}\left(S_{i}+\frac{1}{a_{i}} \log \frac{k_{i}}{k_{j}}\right) .
$$

This is a familiar quota-share treaty, with quotas $q_{j}$ and side-payments $y_{j}(0)$. As $q_{j}$ does not depend on the constants $k_{j}$, the bargaining procedure will only have to determine the amount of the compensations $y_{j}(\mathrm{o})$.

Suppose that the three companies only differ by their attitude towards risk: $a_{1}=.3, a_{2}=.6, a_{3}=.1$, while the other parameters are equal: the reserves equal to 10 , and the total claim amounts are $\Gamma$-distributed, with a mean 1.2 and a variance 1.25 .

The initial utilities are then

$$
\begin{aligned}
& U_{1}\left(x_{1}\right)=3.0778 \\
& U_{2}\left(x_{2}\right)=1.6539 \\
& U_{3}\left(x_{3}\right)=5.8242 .
\end{aligned}
$$

The treaties arising from the merging of two companies are

1. $\{1\} \dot{U}\{2\}:$ Quotas $q_{1}=2 / 3 \quad$ Side payment $y_{1}(0)=-0.6778$

$$
q_{2}=1 / 3
$$

Utilities after reinsurance $U_{1}[\bar{y}(12)]=3.1014$

$$
U_{2}[\bar{y}(12)]=1.6560 ;
$$


2. $\{1\} \dot{U}\{3\}:$ Quotas $q_{1}=1 / 4 \quad$ Side payment $y_{1}(0)=0.7111$

$$
q_{3}=3 / 4
$$

Utilities after reinsurance $U_{1}[\bar{y}(13)]=3.0856$

$$
U_{3}[\bar{y}(13)]=5.8676
$$

3. $\{2\} \dot{U}\{3\}$ : Quotas $q_{2}=.1429$ Side payment $y_{2}(0)=-1.2180$

$$
q_{3}=.8571
$$

Utilities after reinsurance $U_{2}[\bar{y}(23)]=1.6560$

$$
U_{3}[\bar{y}(23)]=5.9599 \text {. }
$$

Adding the third player leads to quotas $q_{1}=2 / 9, q_{2}=1 / 9, q_{3}=2 / 3.3$, being the least risk averse, takes advantage of this to attract a large proportion of its partners' portfolios. As a compensation for its increased liabilities, it will naturally demand a high fixed sum. We obtain the following side payments and utilities.

$$
\begin{aligned}
& \text { Side payments } \\
& \text { 1. }\{12\} \dot{U}\{3\} \\
& y_{1}(0)=.2127 \\
& y_{2}(0)=1.0844 \\
& y_{3}(\mathrm{o})=-1.2971 \\
& \text { Utilities } \\
& \text { 2. }\{13\} \dot{U}\{2\} \quad y_{1}(0)=\quad .2882 \\
& y_{2}(0)=1.2576 \\
& U_{1}(\bar{y})=3.1065 \\
& U_{2}(\bar{y})=1.6565 \\
& U_{3}(\bar{y})=5.8565 \\
& y_{3}(0)=-1.545^{8} \\
& U_{1}(\bar{y})=3.1013 \\
& U_{2}(\bar{y})=1.6554 \\
& U_{3}(\bar{y})=5.9583 \\
& \text { 3. }\{1\} \dot{U}\{23\} \\
& y_{1}(0)=\quad .5356 \\
& y_{2}(0)=1.0890 \\
& U_{1}(\bar{y})=3.0834 \\
& U_{2}(\bar{y})=1.6565 \\
& y_{3}(0)=-1.6264 \\
& U_{3}(\bar{y})=5.9897 .
\end{aligned}
$$

The last company to enter the bargaining has a solid disadvantage.

With the set of distances $D_{1}=\left(d_{12}=1, d_{13}=2, d_{23}=2.5\right)$, the final solution is

$$
\begin{aligned}
& y_{1}(0)=.2627 \\
& U_{1}(\bar{y})=3.1031 \\
& y_{2}(0)=1.1156 \\
& y_{3}(\mathrm{o})=-1.3783 \\
& U_{2}(\bar{y})=1.6565 \\
& U_{3}(\bar{y})=5.8897
\end{aligned}
$$

1 and 2 take advantage of their vicinity to pay as less as possible to 3 . If we suppose that 1 and 3 are the closest friends, i.e. that $D_{2}=\left(d_{12}=2, d_{13}=1\right.$, $d_{23}=2.5$ ), the final treaty is

$$
\begin{aligned}
& y_{1}(0)=.3029 \\
& y_{2}(0)=1.2078 \\
& U_{1}(\bar{y})=3.1003 \\
& y_{3}(0)=-1.5107 \\
& U_{2}(\bar{y})=1.6557 \\
& U_{3}(\bar{y})=5.9438 \text {. }
\end{aligned}
$$

As the initial utilities correspond to side payments of $\left(y_{1}(0)=.6096\right.$, $\left.y_{2}(0)=1.4659, y_{3}(0)=-1.2201\right)$ the final solution achieves the same utility increase as a gain in capital of $(.3469, .3503, .1582)$ for the set $D_{1}$, and of $(.3067, .2581, .2906)$ for $D_{2}$. 


\section{RESOLUTION SCHEME OF ALL FOUR-PERSON GAMES}

1. Treaties for all the sub-sets of two or three players: see $\S 5$.

2. Treaty for the grand coalition. Distances $\left(d_{12}, d_{13}, d_{13}, d_{23}, d_{24}, d_{34}\right)$.

\begin{tabular}{|c|c|c|c|}
\hline$m$ & Configuration & Probability & \\
\hline 4 & $(1,2,3,4)$ & & \\
\hline \multirow[t]{6}{*}{3} & $(12,3,4)$ & $W_{12,3,4}=A / d_{12}^{2}$ & \\
\hline & $(13,2,4)$ & $W_{13,2,4}=A / d_{13}^{2}$ & with $A=$ \\
\hline & $(14,2,3)$ & $W_{14,2,3}=A / d_{14}^{2}$ & 然 \\
\hline & $(1,23,4)$ & $W_{1,23,4}=A / d_{23}^{2}$ & $1+1+1+1$ \\
\hline & $(1,24,3)$ & $W_{1,24,3}=A / d_{24}^{2}$ & $\overline{d_{12}^{2}}+\overline{d_{13}^{2}}+\overline{d_{14}^{2}}+\overline{d_{23}^{2}}+\overline{d_{24}^{2}}+\overline{d_{34}^{2}}$ \\
\hline & $(1,2,34)$ & $W_{1,2,34}=A / d_{34}^{2}$ & \\
\hline
\end{tabular}

\begin{tabular}{|c|c|c|c|c|}
\hline$m$ & Parent & Descendant & Probability & \\
\hline 2 & $\begin{array}{l}(1,23,4) \\
(1,24,3) \\
(1,2,34)\end{array}$ & $\begin{array}{l}(123,4) \\
(124,3) \\
(12,34) \\
(123,4) \\
(134,2) \\
(13,24) \\
(124,3) \\
(134,2) \\
(14,23) \\
(123,4) \\
(1,234) \\
(14,23) \\
(124,3) \\
(1,234) \\
(13,24) \\
(1,234) \\
(134,2) \\
(12,34)\end{array}$ & $\begin{array}{l}\left.W_{123,4}\right|_{12,3,4}=B / d_{12,3}^{2} \\
\left.W_{124,3}\right|_{12,3,4}=B / d_{12,4}^{2} \\
\left.W_{12,34}\right|_{12,3,4}=B / d_{34}^{2} \\
\left.W_{123,4}\right|_{13,2,4}=C / d_{13,2}^{2} \\
\left.W_{134,2}\right|_{13,2,4}=C / d_{13,4}^{2} \\
\left.W_{13,24}\right|_{13,2,4}=C / d_{24}^{2} \\
\left.W_{124,3}\right|_{14,2,3}=D / d_{14,2}^{2} \\
\left.W_{134,2}\right|_{14,2,3}=D / d_{14,3}^{*} \\
\left.W_{14,23}\right|_{14,2,3}=D / d_{23}^{2} \\
\left.W_{123,4}\right|_{1,23,4}=E / d_{12,3}^{2} \\
\left.W_{1,234}\right|_{1,23,4}=E / d_{29,4}^{2} \\
\left.W_{14,23}\right|_{1,23,4}=E / d_{14}^{2} \\
\left.W_{124,3}\right|_{1,24,3}=F / d_{1,24}^{2} \\
\left.W_{1,234}\right|_{1,24,3}=F / d_{24,3}^{2} \\
\left.W_{13,24}\right|_{1,24,3} ^{2}=F / d_{13}^{2} \\
\left.W_{1,234}\right|_{1,2,34}=G / d_{2,34}^{2} \\
\left.W_{134,2}\right|_{1,2,34}=G / d_{1,34}^{2} \\
\left.W_{12,34}\right|_{1,2,34}=G / d_{12}^{2}\end{array}$ & $\begin{array}{l}\text { with } B= \\
\left(\frac{1}{d_{1,3}^{2}}+\frac{1}{d_{12,4}^{2}}+\frac{1}{d_{34}^{2}}\right)^{-1} \\
\text { with } C= \\
\left(\frac{1}{d_{13,2}^{2}}+\frac{1}{d_{13,4}^{2}}+\frac{1}{d_{24}^{2}}\right)^{-1} \\
\text { with } D= \\
\left(\frac{1}{d_{14,2}^{2}}+\frac{1}{d_{14,3}^{2}}+\frac{1}{d_{23}^{2}}\right)^{-1} \\
\text { with } E= \\
\left(\frac{1}{d_{12,3}^{2}}+\frac{1}{d_{23,4}^{2}}+\frac{1}{d_{14}^{2}}\right)^{-1} \\
\text { with } F= \\
\left(\frac{1}{d_{1,24}^{2}}+\frac{1}{d_{24,3}^{2}}+\frac{1}{d_{13}^{2}}\right)^{-1} \\
\text { with } G= \\
\left(\frac{1}{d_{2,34}^{2}}+\frac{1}{d_{1,34}^{2}}+\frac{1}{d_{12}^{2}}\right)^{-1}\end{array}$ \\
\hline
\end{tabular}

\begin{tabular}{lll}
\hline \hline$m$ & Configuration & Treaty \\
\hline 1 & $W_{123,4}=A / d_{12}^{2} B / d_{1,3}^{2}+A / d_{13}^{2} C / d_{13,2}^{2}+A / d_{23}^{2} E / d_{1,23}^{2}=W_{123,4}$ & $\bar{y}\left(123 \dot{U}_{4}\right)$ \\
& $W_{124,3}=A / d_{12}^{2} B / d_{12,4}^{2}+A / d_{14}^{2} D / d_{14,2}^{2}+A / d_{24}^{2} F / d_{1,24}^{2}=W_{124,3}$ & $\bar{y}\left(124 \dot{U}_{3}\right)$ \\
& $W_{134,2}=A / d_{13}^{2} C / d_{13,4}^{2}+A / d_{14}^{2} D / d_{14,3}^{2}+A / d_{34}^{2} G / d_{1,34}^{2}=W_{134,2}$ & $\bar{y}\left(134 \dot{U}_{2}\right)$ \\
$W_{1,234}=A / d_{23}^{2} E / d_{2,4}^{2}+A / d_{24}^{2} F / d_{24,3}^{2}+A / d_{34}^{2} G / d_{2,34}^{2}=W_{1,234}$ & $\bar{y}\left(1 \dot{U}_{234}\right)$ \\
$W_{12,34}=A / d_{12}^{2} B / d_{34}^{2}+A / d_{34}^{2} G / d_{12}^{2}=W_{12 \dot{U} 34}$ & $\bar{y}\left(12 \dot{U}_{34}\right)$ \\
$W_{13,24}=A / d_{12}^{2} C / d_{34}^{2}+A / d_{24}^{2} F / d_{13}^{2}=W_{13 \dot{U} 24}$ & $\bar{y}\left(13 \dot{U}_{24}\right)$ \\
$W_{14,23}=A / d_{14}^{2} D / d_{23}^{2}+A / d_{23}^{2} E / d_{14}^{2}=W_{14 \dot{U} 23}$ & $\bar{y}\left(14 \dot{U}_{23}\right)$ \\
\hline
\end{tabular}


Example 4. The homogeneous weighted majority game $(3 ; 2,1,1,1)_{h}$.

This four-person game, a simplification of the game "Me and my Aunt" was studied by Owen [9] in his generalization of the Shapley value. The strongest player, 1, possesses two votes, while each of his opponents has only one. As three votes are required to win the game, the only winning coalitions are

(i) 1 and one, two or all three of his partners,

(ii) 234 .

The game is however complicated by the fact that players 1 and 2 are parents; in fact, 1 is 2 's aunt. Since we only want to study the influence of this relationship, we can set $d_{12}=1$ and all the other distances equal to 2 .

\begin{tabular}{|c|c|c|c|c|c|c|}
\hline \multirow{2}{*}{$\begin{array}{c}\text { Coalition formation } \\
{ }_{123} \dot{U}_{4}\end{array}$} & \multicolumn{2}{|l|}{ Weight } & \multicolumn{4}{|c|}{ Utility } \\
\hline & $W_{123,4}=.2527$ & & $(.4722$, & .3889 & .1389 & .0 \\
\hline $124 \dot{U}_{3}$ & $W_{124,3}=.2527$ & & $(.4722$ & .3889 & $.0 \quad$, & $.13^{89)}$ \\
\hline${ }_{134} \dot{U}_{2}$ & $W_{134,2}=.0774$ & & $(.4444$, & .o , & .2778 & $\left..277^{8}\right)$ \\
\hline${ }_{1} \dot{U}_{234}$ & $W_{1,234}=.0774$ & & (.o , & .3333 & .3333 & $.3333)$ \\
\hline${ }_{12} \dot{U}_{34}$ & $W_{12,34}=.2222$ & & $(.5$ & $.5 \quad$ & $.0 \quad$ & $.0 \quad)$ \\
\hline${ }_{13} \dot{U} 24$ & $W_{13,24}=.0588$ & & $(.5$ & .0 & .5 & .0 \\
\hline \multirow[t]{2}{*}{${ }_{14} \dot{U}_{23}$} & $W_{14,23}=.0588$ & & $(.5$ & .0 & $.0 \quad$ & .5 \\
\hline & & Value & $\left(.443^{\circ}\right.$ & .3334 & .1118 & $.1118)$ \\
\hline
\end{tabular}

The solution converges towards $\left(\begin{array}{llllll}.5 & .5 & , & .0 & , & .0\end{array}\right)$ when $d_{12} \rightarrow 0$. Owen's modified version of Shapley's value tends to $(2 / 3,1 / 3$, $o, o, o$ ) in this case (see discussion of $\S 7$ ).

\section{A FIVE-PERSON GAME}

Example 5. Me and my Aunt.

This is the original game introduced by Davis and Maschler, perhaps the most celebrated game of the theory (see [2] for an interesting discussion of the game). It is in fact the homogeneous weighted majority game $(4 ; 3,1,1,1,1)_{h}$ with the addition that player 1 (my aunt) and player 2 (me) "in principle" agree to form a coalition.

The Shapley value is

The kernel, the nucleolus and the $\left(\begin{array}{lllll}.6, & .1, & .1, & .1, & .1\end{array}\right)$

$(3 / 7, \quad 1 / 7, \quad 1 / 7, \quad 1 / 7, \quad 1 / 7)$

Nash-Lemaire value agree on a

division proportional to the weights $=(.4286, .1428, .1428, .1428, .1428)$

Most of the discussions among the game theorists in fact center on the words "in principle": the problem is phrased in an asymmetric fashion, whereas it is symmetric in terms of payoffs to coalitions. One way to capture into the model the preferences between 1 and 2 is to introduce some external feature, like our "affinities", independently of the characteristic function. 
The computation of the weighted value, assuming that $d_{12}=1$ and $d_{i j}=2$ $\forall(i j) \neq(12)$ becomes rather lengthy. The solution is

$$
(.4472, .2849, .0893, .0893, .0893)
$$

and favour the nephew more than his aunt. The payoff vector converges towards $(.5, .5, .0, .0, .0)$ when $d_{12} \rightarrow 0$, a division that we feel more intuitive than Owen's limiting value $(.75, .25, .0, .0, .0)$. As a matter of fact, we think that, if 2 knows that his aunt feels compelled to agree with him and that the other players are consequently irrelevant, he should be able to "extract" $\frac{1}{2}$ from her. If the blood ties are strong enough, no other partnership is thinkable, and any threat of the aunt to negotiate with somebody else will not be credible: the asymmetry between 1 and 2 disappears and the equal division seems the only fair payoff.

Remark that the limit value does not depend on the particular choice of the rule $R$.

Note that the bargaining set for the configuration $(12,345)$ grants player $1 \mathrm{a}$ payoff in the interval [.50.75] (it of course does not introduce any consanguinity in the problem). Our value thus stands at one end of this interval (the more generous towards the weaker player), Owen's generalization at the other end.

The different concepts of value attempt to be good predictors of the actual outcomes of negotiations. It is thus always interesting to compare the values with experimental data. "Me and my Aunt" has been effectively played 12 times under the direction of Selten and Schuster [11] (no preference relationship was introduced in the experiments). The game ended 8 times with a coalition between 1 and 2 , with a payoff to 1 always inferior than .75 . The division $(.75, .25, .0, .0, .0)$ appeared twice during bargaining, but the stronger player was never able to protect his share and the coalition broke off. The average payoff was .4668 to $1, .1333$ to the other players, a division that seems consistent with the predictions of the kernel and our unweighted value.

The facts that:

(i) the average gain of 1 was well under the figure predicted by the Shapley value;

(ii) even without affinities, 1 was never able to force a gain of .75 ,

naturally corroborates the idea that the Shapley value (or modified value) seems to be too generous towards the stronger players, by overlooking the threat possibilities of the weaker players.

It can besides be shown that, for $n>2$, our value will always award more to the weaker players than Shapley's value. It is due to the fact that, if one accepts Shapley's axioms, the pivotal player becomes all of his admission value, while the axioms of $\S 2$ have the effects by (3) of sharing this quantity between the members of the coalition according to their respective strengths. 


\section{REFERENCES}

[1] Bühlmann, H. (1970). Mathematical methods in risk theory, Berlin.

[2] Davis, M. and Maschler M. (1965). The kernel of a cooperative game, Naval Research Logistics Quavterly, 12, 223-259.

[3] Gerber, H. (1974). On additive premium calculation principles, Astin Bulletin, 7, $215^{-222}$

[4] Gerber, H. (1974). On iterative premium calculation principles, Mitteilungen der Vereinigung Schweizerischer Versicherungsmathematiker, 74, 163-172.

[5] Lemaire, J. (1973). Optimalité d'un contrat d'échange de risques entre assureurs, Cahiers du C.E.R.O. Bruxelles, 15, 139-156.

[6] Lemaike, J. (1973). A new value for games without transferable utilities, International Journal of Game Theory, 2, 205-213.

[7] Lemaire, J. (1977). Echange de risques entre assureurs et théorie des jeux, Astin Bulletin, 9, 155-179.

[8] NASH, J. (1950). The bargaining problem, Econometrica, 18, 155-162.

[9] Owen, G. (1971). Political games, Naval Research Logistics Quarterly, 18, 345-355.

[10] Owen, G. (1972). Values of games without side payments, International Journal of Game Theory, 1, 95-110.

[11] Selten, R. and K. Schuster (1968). Psychological variables and coalition forming behavior, Proc. of the conference of the IEA (Smolenice), London, 221-246.

[12] Shapley, I. S. (1953). A value for n-person games, Annals of Maths. Studies, 28, 307-318, Princeton. 